BIOSYNTHESIS

\section{Served with a twist}

of the tripartite complex. Two of the hits from the screen and a related compound (C3) inhibited HIV-1 replication in vitro. The results of a competition assay validated that C3 targets the Tat-CycT1 interface. C3 specifically inhibited Tat-mediated HIV-1 transcription and also suppressed Tatinduced phosphorylation of RNAPII, which is essential for its activity on HIV-1 transcripts. C3-based compounds may prove useful in combination with anti-HIV-1 drugs that act by other mechanisms.

\title{
PLANT SIGNALING
}

\section{Flowers from a sweetheart Science 339, 704-707 (2013)} conformational searching by the constrained sequence. The authors therefore conclude that the thioesterases impart different structures on the peptide during cyclization rather than display different sequence preferences. Tests of the two populations of occidiofungin against several Candida species demonstrated that the wild-type pool was substantially more effective than the knockout sample at inhibiting colony-forming units, though the minimum inhibitory concentration was decreased by only a factor of two; whether the OcfN-derived structure is more active or the two are synergistic remains unknown.

\section{METABOLISM}

\section{RNA talks back}

PLoS Biol., published online 12 February 2013; doi:10.1371/journal.pbio.1001482

Metabolic enzymes are often expressed constitutively, even though their activities may only be needed under specific conditions. Thus, identifying mechanisms by which these enzymes are regulated is an important challenge. A subset of metabolic enzymes has been found to bind RNA, but the functional implications of these interactions remain unclear. To explore this question, Bohne et al. identify a protein that binds the $p s b A$ mRNA, which encodes a subunit of photosystem II, as dihydrolipoamide acetyltransferase (DLA2), an enzyme in the chloroplast pyruvate dehydrogenase complex that provides acetyl-CoA for fatty acid synthesis. DLA2 assembles with the RNA into a high-molecular-weight complex in a light- and acetate-dependent manner, with the complexes localized to the 'chloroplast translation membrane', a translational site within the chloroplast. $p s b A$ expression but not transcription was reduced in the absence of DLA2, again depending on the availability of light and acetate. The authors also tested whether a reciprocal interaction existed, in which $p s b A$ mRNA regulated DLA2 function, and observed a substantial reduction in acetyl-CoA production in the presence of mRNA. This interference could be the result of direct competition, as the putative RNA-binding site in DLA2 overlaps with the region known to mediate assembly into the chloroplast pyruvate dehydrogenase complex. These results not only demonstrate a new mechanism for DLA2 regulation but also point to a broader crosstalk between fatty acid and protein synthesis in the chloroplast. $\quad C G$

\section{DRUG DISCOVERY}

\section{Hits for Tat}

Antimicrob. Agents Chemother. 57, 1323-1331 (2013)

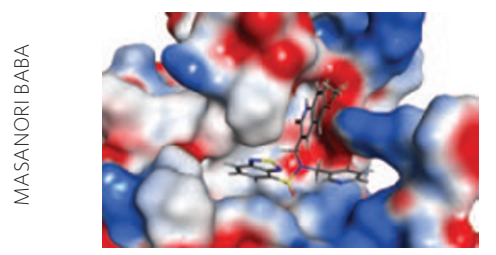

HIV-1 has an RNA genome that is generated by the transcription of its proviral DNA through a series of events starting from the viral transcriptional activator Tat. Tat interacts specifically with host cyclin T1 (CycT1), a component of the RNAPII transcription elongator factor complex, which enhances binding of the complex to cis-acting transactivation-responsive (TAR) RNA at the $5^{\prime}$ end of nascent HIV- 1 transcripts. RNAPII then begins the elongation of HIV-1 transcripts. The interactions between the elongation factor complex, Tat and TAR RNA are therefore crucial for replication of the HIV-1 genome. Hamasaki et al. searched for compounds that could bind the Tat/TAR RNA recognition motif (TRM) of CycT1 by in silico screening of 3 million compounds. The authors docked members of the library to a TRM identified in a homology model

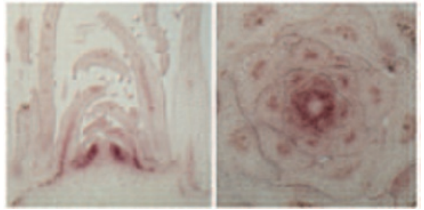

To guarantee reproductive success, plants regulate flowering to ensure that it occurs under optimal environmental and metabolic conditions. Leaves sense a lengthening photoperiod, which triggers production of FLOWERING LOCUS T (FT), a mobile florigen that induces flower development in the shoot apical meristem (SAM). Plants also sense whether they have sufficient energy resources for flowering, and mutations in genes involved in carbohydrate metabolism delay flowering. Wahl et al. now show that trehalose-6-phosphate (T6P) regulates several flowering pathways in Arabidopsis thaliana. Knockdown of TREHALOSE-6PHOSPHATE SYNTHASE 1 (TPS1), the enzyme that synthesizes T6P, reduces T6P cellular concentrations and delays flowering. In wild-type plants, T6P concentrations correlate with carbohydrate abundance and undergo cycling with a diurnal period. However, in TPS1 mutants, the expression of FT is blocked, suggesting that TPS1 acts upstream of FT in regulating the photoperiod pathway in leaves. T6P also controls floral induction directly in the SAM. Microarray analysis of TPS1 mutants revealed that T6P partially regulates SQUAMOSA PROMOTER BINDING PROTEIN-LIKE (SPL) proteins, known to be involved in age-dependent induction of flowering in the SAM. Though further research will be required to establish the molecular mechanism of T6P action in plants, the current study highlights an additional role of trehalose derivatives in regulating biological systems.

Written by Mirella Bucci, Amy Donner, Joshua M. Finkelstein, Catherine Goodman \& Terry L. Sheppard 